\title{
MODELOS DE TRANSFERÊNCIA DE METAL PESADO NA CANA-DE-AÇÚCAR ADUBADA COM COMPOSTO DE LIXO URBANO
}

\author{
FÁBIO C. DA SILVA ${ }^{1}$, ALESSANDRA F. BERGAMASCO ${ }^{2}$, LAERCIO L. VENDITE ${ }^{3}$
}

RESUMO: A pesquisa aborda a reciclagem do composto de lixo urbano (CLU) como fertilizante alternativo na cana-de-açúcar e como solução social e ambiental ao acúmulo de resíduos sólidos nos centros urbanos. Utilizou-se da modelagem matemática para conhecer a dinâmica dos metais pesados, visando ao estabelecimento de critérios e procedimentos para o uso seguro do CLU, limitado pela quantidade desses elementos. Foram construídos modelos compartimentais a partir de dados de experimentos em condições controladas e parcialmente validados com dados de campo. Esse modelo descreveu a transferência de metais pesados no sistema solo-raiz-parte aérea para a cana-de-açúcar. Pôde-se concluir, pelas condições desta pesquisa, que o metal mais preocupante foi o níquel, pois demora, aproximadamente, três anos para ser atenuado no solo e chega em maior quantidade na parte aérea. Quanto aos fatores argila, óxidos e $\mathrm{pH}$ do solo, notou-se que, nos solos de maior poder tampão, a passagem da maioria dos metais foi mais lenta. Esse modelo pode tornarse importante aliado na definição de leis de utilização do CLU, visando à não-contaminação ambiental, redução no acúmulo de lixo e de custos de produção.

PALAVRAS-CHAVE: composto orgânico, modelo matemático, meio ambiente.

\section{HEAVY METAL TRANSFERENCE MODELS IN SUGARCANE FERTILISED WITH URBAN WASTE COMPOST}

\begin{abstract}
The research approaches recycling of urban waste compost (UWC) as an alternative fertilizer for sugarcane crop and as a social and environmental solution to the solids residuals growth in urban centers. A mathematical model was used in order to know the metal dynamics as decision support tool, aiming to establish of criteria and procedures for UWC's safe use, limited by the amount of heavy metal. A compartmental model was developed from experimental data in controlled conditions and partially checked with field data. This model described the heavy metal transference in the system soil-root-aerial portion of sugarcane plants and concluded that nickel was metal to be concern, since it takes approximately three years to be attenuated in the soil, reaching the aerial portions of the plant at high concentrations. Regarding factors such as clay content, oxide level and soil $\mathrm{pH}$, it was observed that for soil with higher buffering capacity, the transfer of the majority of the metals was slower. This model may become an important tool for the attainment of laws regarding the UWC use, aiming to reduce environment contamination the waste accumulation and production costs.
\end{abstract}

KEYWORDS: urban waste compost, mathematical model, environment.

\section{INTRODUÇÃO}

Os lixos urbanos, quando acumulados, causam diversos prejuízos à população e ao meio ambiente, tornando-se um problema governamental que está agravando-se devido ao crescimento populacional e à rápida urbanização. Uma maneira de solucionar esse problema é mediante a compostagem do lixo domiciliar, do qual se origina o composto de lixo urbano (CLU), sendo o uso na agricultura a melhor opção para sua disposição final como fonte de nutrientes $(\mathrm{P}, \mathrm{K}, \mathrm{Ca}, \mathrm{Mg}, \mathrm{Cu}$ e Zn) e condicionador das propriedades físicas (ALVES, 1996) e químicas de solos ácidos (SILVA

\footnotetext{
${ }^{1}$ Engo Agrônomo e Florestal, Doutor, Embrapa Informática Agropecuária, Campinas - SP, Pós-doutorando no Departamento de Produção Vegetal na ESALQ/USP, fcesar@ cnptia.embrapa.br

${ }^{2}$ Zootecnia, Mestre, Bolsista Fapesp, Faculdade de Engenharia Agrícola - Unicamp. afb@agr.unicamp.br

${ }^{3}$ Matemático, Prof. Associado, IMECC/ UNICAMP, Campinas - SP. vendite@ime.unicamp.br

Recebido pelo Conselho Editorial em: 15-6-2005
}

Aprovado pelo Conselho Editorial em: 14-11-2006 
et al., 2002), refletindo em aumentos na capacidade de troca catiônica (CTC) e também na saturação por bases (V\%). Porém, deve-se atentar para o fato de que esse CLU pode vincular metais pesados, fator limitante de seu uso e que requer controle no produto (CLU) para fins de comercialização e também as formas de sua aplicação no solo (SILVA et al., 2002; ENVIRONMENTAL PROTETION AGENCY - EPA, 1995), assim como os valores orientados máximos para a proteção da qualidade de solos no Estado de São Paulo, visando à prevenção da contaminação ambiental (CETESB, 2001).

SILVA et al. (2002) utilizaram os resultados de experimentos de incubação solo/CLU, nos quais se estudou a disponibilidade de alguns metais do CLU aplicados aos solos no decorrer do tempo e obtiveram modelo de decaimento da disponibilidade do metal no solo. Em vários solos, o tempo de decaimento desse metal é bem longo, isto é, a redução de metade do metal disponível às plantas no solo será de seis a 12 meses, tornando o uso do CLU mais vulnerável nesses solos, necessitando de cuidados maiores e contínuo monitoramento.

Na revisão de BAILEY et al. (1999), elucida-se a transferência de metais pesados no sistema solo-planta-resíduo, discutindo-se a evolução científica para a obtenção de modelos para metais, que descrevam fenômenos e que possam predizer os riscos ambientais envolvidos na utilização de efluentes e de resíduos sólidos em solos. Os modelos matemáticos baseados em especiação iônica em solução, na adsorção e na difusão de íons no solo, têm-se destacado, que são influenciados pelas formas, quantidade e mineralogia da argila e os teores de outros elementos no meio (MEERS et al., 2006). O efeito tóxico de metal é diretamente relacionado a sua concentração em solução do solo, a qual é condicionada pelas características da sua fração sólida: capacidade de troca iônica, matéria orgânica e $\mathrm{pH}$, que reflete nos processos de precipitação, de adsorção e de complexação (VIJVER et al., 2003). A abordagem na modelagem seria focar o comportamento de um metal em solução e suas interações como os ligantes orgânicos e inorgânicos existentes no solo, cujos cálculos são realizados pelo software Minteq (TIPPING, 1998).

Existem estudos específicos para passagem de cada metal para as raízes e parte aérea das culturas, como BENAVIDES et al. (2005), que resumem o conhecimento na área de respostas de plantas ao cádmio, incluindo mecanismos de homeostase e o estresse oxidativo de cádmio, absorção, transporte e acúmulo. Os modelos de passagem de metais para as plantas, em função dos teores dos metais no solo, de acordo com McBRIDE (1995), seriam três os mais conhecidos na literatura: (a) linear ou associação de duas equações lineares (com constantes de partição do metal na planta), que tem sido usado para o chumbo; (b) modelo de platô de saturação, o mais utilizado, e (c) absorção de Langmuir.

Devido à importância do tema, avaliaram-se os efeitos da aplicação do composto de lixo enriquecido ou não com metais pesados no cultivo da cana-de-açúcar, em especial a dinâmica dos metais pesados no sistema solo-raiz-parte aérea, utilizando-se de modelos matemáticos que podem auxiliar na minimização dos riscos ambientais e proporcionar a utilização sustentável do composto de lixo urbano, sem prejudicar o ambiente, devido ao fato de visualizarem, por simulação numérica, as melhores opções de taxas, áreas e uso do composto sobre a produtividade da cultura de cana-de-açúcar.

\section{MATERIAL E MÉTODOS}

Foram realizados dois experimentos com cana-de-açúcar (SILVA et al., 2000ab), adubando-a com CLU, em condições controladas na Embrapa Solos e, em ambos os estudos, os solos de cada um foram separados em classes de acordo com os teores de óxido de $\mathrm{Fe}$ e $\mathrm{Al}$, teor de argila e $\mathrm{pH}$, com o objetivo do estudo da interferência desses fatores nas concentrações de metais pesados e simular modelos dentro de variáveis homogêneas. As classes de solos foram: Classe A, para solos com argila $<35 \%$, óxidos $\mathrm{Fe} / \mathrm{Al}<12 \%$ e $\mathrm{pH}$ água $<5,3$ e Classe $\mathrm{B}$, solos com argila $>35 \%$, óxidos $\mathrm{Fe} / \mathrm{Al}>12 \%$ e pH água $>5,3$. 


\section{Experimento 1: Estudo da disponibilidade de metais pesados no tempo}

Esse experimento foi conduzido por incubação de diferentes solos com CLU, realizado na Embrapa/RJ, onde foram coletadas amostras de cinco tipos de solos (Latossolo Vermelho - LV, Planossolo - S, Chernossolo - M, Luvissolo - T e Nitossolo - N), em duas profundidades (0-20 e 20-40 cm), incubadas com quatro diferentes doses de CLU $\left(0 ; 25 ; 50\right.$ e $100 \mathrm{Mg} \mathrm{ha}^{-1}$ em base úmida), em cinco períodos de incubação $(0 ; 16 ; 32 ; 64$ e 100 dias). $\mathrm{O}$ experimento foi realizado em delineamento inteiramente casualizado, em esquema fatorial $5 \times 2 \times 4 \times 5$, totalizando 200 subtratamentos. A montagem do experimento foi conduzida em laboratório, com controle do teor de água, por meio de diferença de peso.

Para a comparação química de cada tratamento, foram realizadas as seguintes determinações laboratoriais (SILVA, 1999): $\mathrm{pH}$ em $\mathrm{CaCl}_{2}\left(0,01 \mathrm{~mol} \mathrm{~L}^{-1}\right)$; teor disponível de metais pesados (Cd, $\mathrm{Ni}$ e $\mathrm{Pb}$ ), mais $\mathrm{Ca}$ e $\mathrm{Mg}$, usando-se o extrator Mehlich 1 e determinação feita por espectrometria de emissão ótica em plasma de argônio induzido (ICP-OES); e teor total de metais pesados (Cd, $\mathrm{Ni}$ e $\mathrm{Pb}$ ), e $\mathrm{Ca}$ e $\mathrm{Mg}$, com extração com água régia e determinação por ICP-OES.

Os resultados obtidos com os experimentos em vasos foram os teores disponíveis e totais dos metais $\mathrm{Cd}, \mathrm{Cu}, \mathrm{Ni}$ e $\mathrm{Pb}$, nos cinco solos e nas duas profundidades, extraídos nos cinco tempos de incubação do CLU e com três repetições, cujas informações foram submetidas à análise estatística, utilizando o programa estatístico SAS (STATISTICAL ANALYSIS SYSTEM, 1990).

Com os modelos de regressão obtidos no estudo da interferência do tempo de incubação sobre a disponibilidade, foram obtidos valores utilizados na determinação da taxa de decaimento do metal no tempo, mediante o valor " $b$ " do modelo linear de regressão, no qual: $\mathrm{X}=$ tempo de incubação e $\mathrm{Y}=$ teor de metal no solo $(Y=a+b X)$, cuja taxa é um dos parâmetros do modelo de transferência de metal pesado no sistema. Foi também realizado um estudo de simulação para se chegar ao tempo em que o metal atinge metade de seu teor disponível no momento da incubação (meia-vida do metal), em que o modelo exponencial foi ajustado também, que é importante para o conhecimento do tempo necessário para nova aplicação do CLU.

\section{Experimento 2: Estudo da dinâmica de metais pesados no sistema solo-planta}

O experimento 2 foi conduzido em casa de vegetação e no laboratório da Embrapa/RJ, em condição controlada, onde os tratamentos consistiram de quatro solos (LV, S, M e T) incubados com CLU enriquecido com cinco níveis de metais pesados: $\mathrm{Cd}\left(0 ; 2 ; 4 ; 6\right.$ e $\left.8 \mathrm{mg} \mathrm{kg}^{-1}\right)$; $\mathrm{Ni}$ e $\mathrm{Cu}(0$; 50; 100; 200 e $\left.300 \mathrm{mg} \mathrm{kg}^{-1}\right)$ e $\mathrm{Pb}\left(0 ; 100 ; 200 ; 300\right.$ e $\left.400 \mathrm{mg} \mathrm{kg}^{-1}\right)$. Com os resultados dos experimentos em vasos de cana-de-açúcar, foram feitas análises de regressão, visando a estimar a transferência do metal pesado nas concentrações de metais no sistema solo-cana, assim como coeficientes de passagem e comportamento do metal nessa transferência, podendo, assim, oferecer subsídio para a formulação de normas de uso do composto de lixo para a cana-de-açúcar, estipulando-se limites de concentrações de metais nos compostos e a serem adicionados anualmente na área, prevendo-se níveis de fertilização de CLU que não ultrapassem esses limites.

\section{Legislação sobre quantidade de metais pesados nos solos}

Foram estabelecidos pela CETESB (2001) dois tipos de valores-limites para os teores totais dos metais pesados (Tabela 1), com base em revisão bibliográfica sobre fitotoxidade. O primeiro é o valor de alerta, que indica possível alteração da qualidade natural dos solos onde será utilizado em caráter preventivo; o segundo é o valor de intervenção, que indica o limite de contaminação do solo, acima do qual existe risco potencial à saúde humana, e será utilizado em caráter corretivo no gerenciamento de áreas contaminadas, cujo valor excedido requer alguma forma de intervenção para não prejudicar a qualidade da água. Esses valores foram utilizados como parâmetros para comparação com os resultados de quantidades de metais máximos nos solos e na planta, obtidos pelo modelo matemático de transferência de metais pesados, visando à não-contaminação ambiental. 
TABELA 1. Valores-limites de teores totais de metais pesados em solo e na planta, em $\mathrm{mg} \mathrm{kg}^{-1}$.

\begin{tabular}{|c|c|c|c|c|c|c|c|}
\hline \multirow[b]{2}{*}{ Metal } & \multicolumn{5}{|c|}{ 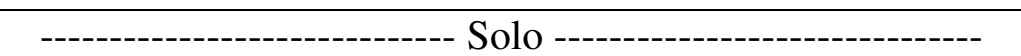 } & \multicolumn{2}{|c|}{------ Planta ----- } \\
\hline & Usual $^{1}$ & $\begin{array}{c}\text { CETESB } \\
\text { Alerta }^{4}\end{array}$ & Variação $^{1}$ & $\begin{array}{c}\text { Nível } \\
\text { Crítico }^{2}\end{array}$ & $\begin{array}{c}\text { CETESB } \\
\text { Intervenção }\end{array}$ & $\begin{array}{l}\text { Intervalo de } \\
\text { Variação }{ }^{1}\end{array}$ & Toxidez $^{3}$ \\
\hline Co & 8 & 25 & $1-65$ & $25-50$ & 40 & $0,02-0,5$ & - \\
\hline $\mathrm{Ni}$ & 40 & 30 & $10-1000$ & 100 & 50 & $0,02-5$ & $10-100$ \\
\hline $\mathrm{Cu}$ & 20 & 60 & $2-100$ & $60-125$ & 100 & $4-15$ & $20-100$ \\
\hline $\mathrm{Zn}$ & 50 & 300 & $10-300$ & $70-400$ & 500 & $8-15$ & $100-400$ \\
\hline $\mathrm{Pb}$ & & 100 & & & 200 & & \\
\hline $\mathrm{Cd}$ & & 3 & & & 10 & & \\
\hline
\end{tabular}

Fonte: ${ }^{1}$ ADRIANO, 1986; ${ }^{2}$ KABATAS-PENDIAS \& PENDIAS, $1985 ;{ }^{3}$ Ponto Inicial fitotoxidade (phytotoxicity threshold - PT 10 e 20\%); ${ }^{4}$ CETESB (2001) - valores orientadores para proteção da qualidade de solos e água no Estado de São Paulo: Alerta e Intervenção (www.cetesb.sp.gov.br).

\section{Modelagem da transferência de metal pesado no sistema solo-planta}

Na modelagem matemática do sistema solo-planta, construiu-se um modelo de transferência de metais pesados, com base nos modelos compartimentais proposto por BASSANEZI \& FERREIRA (1988). Considerou-se um simples modelo determinístico de equações diferenciais lineares para descrever a dinâmica da disponibilidade de cada metal $(\mathrm{Cd}, \mathrm{Cu}, \mathrm{Ni}$ e $\mathrm{Pb})$ no solo e o grau de absorção desse metal pela raiz. Para isso, estabeleceu-se um fluxograma de transferência dos metais pesados aplicado na forma de composto de lixo (CLU) no sistema solo-raiz-parte aérea, considerados três compartimentos (Figura 1):
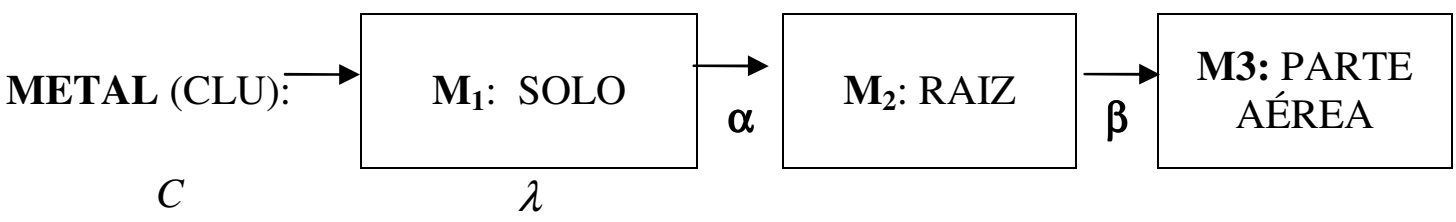

FIGURA 1. Fluxograma da passagem do metal nos compartimentos do sistema solo-raiz-parte aérea da cana-de-açúcar.

Tem-se que : i) compartimento $\mathrm{M}_{1}$ que denota o solo; ii) compartimento $M_{2}$ que denota a raiz, e iii) compartimento $M_{3}$ que denota a parte aérea. Portanto, o modelo proposto baseou-se nas seguintes proposições [eqs. (1), (2) e (3)]:

$$
\begin{aligned}
\frac{\mathrm{dM}_{1}}{\mathrm{dt}} & =-\lambda \mathrm{M}_{1}-\alpha \mathrm{M}_{1} \\
\frac{\mathrm{dM}_{2}}{\mathrm{dt}} & =-\beta \mathrm{M}_{2}+\alpha \mathrm{M}_{1} \\
\frac{\mathrm{dM}}{\mathrm{dt}} & =+\beta \mathrm{M}_{2}
\end{aligned}
$$

em que,

$\mathrm{M}$ - Fonte de metal pesado (CLU);

$\mathrm{M}_{1}$ - concentração do metal pesado no solo no instante t;

$\mathrm{M}_{2}$ - concentração do metal pesado na raiz no instante $\mathrm{t}$;

$\mathrm{M}_{3}$ - concentração do metal pesado na parte aérea da cana-de-açúcar no instante t;

$\lambda$ - velocidade de decaimento do metal pesado (taxa constante);

$\alpha$ - taxa de absorção do metal do solo pela raiz (taxa de absorção da raiz);

$\beta$ - taxa de translocação do metal da raiz à parte aérea (proporcional à taxa de absorção), e $\mathrm{t}$ - tempo. 
As condições iniciais: $\mathrm{M}_{1}(0)=\mathrm{C}, \mathrm{M}_{2}(0)=0$ e $\mathrm{M}_{3}(0)=0$, em que $\mathrm{C}=$ composto de lixo enriquecido com metal pesado $\left(\mathrm{mg} \mathrm{kg}^{-1}\right)$. A eq.(1) é independente, então encontramos a solução exponencial imediata $M_{1}(t)$ dada pela relação (4); a eq.(2) é homogênea $\frac{d M_{2}}{d t}=-\beta M_{2}+\alpha M_{1}$, na qual já conhecemos $M_{1}(t)$ de (4) e, sendo assim, encontramos a relação (5) para $M_{2}(t)$ e analogamente, substituindo o valor de $\mathrm{M}_{2}(\mathrm{t})$ na eq.(3), encontramos a relação (6). Sendo assim, encontramos os seguintes valores para $\mathrm{M}_{1}(\mathrm{t}), \mathrm{M}_{2}(\mathrm{t})$ e $\mathrm{M}_{3}(\mathrm{t})$ :

$$
\begin{aligned}
& \text { Solução 1: } M_{1}(t)=C \mathrm{e}^{-(\lambda+\alpha) t} \\
& \text { Solução 2: } M_{2}(t)=\frac{\alpha C}{\beta-(\lambda+\alpha)}\left(\mathrm{e}^{-(\lambda+\alpha) t}-\mathrm{e}^{-\beta t}\right) \\
& \text { Solução 3: } M_{3}(\mathrm{t})=\frac{\alpha \beta C}{\beta-(\lambda+\alpha)}\left(\frac{1}{\beta} \mathrm{e}^{-\beta \mathrm{t}}-\frac{1}{1+\alpha} \mathrm{e}^{-(\lambda+\alpha) t}\right)+\frac{\alpha C}{\lambda+\alpha}
\end{aligned}
$$

Para a estimativa dos parâmetros do modelo proposto $(\lambda, \alpha$ e $\beta$ ), foram utilizados os dados reais obtidos nos experimentos 1 e 2 , realizando-se ajuste não-linear pelo procedimento PROC NLIN do SAS aos modelos compartimentais, realizando-se interações até a convergência dos parâmetros.

Para o início das interações, é necessária uma estimativa dos parâmetros envolvidos no modelo $(\lambda, \alpha$ e $\beta$ ), obtidos pelas análises de regressão feitas anteriormente aos dados do Experimento 2, em que o valor do parâmetro $b$ do modelo linear $\mathrm{M}_{2}=\mathrm{a}+\mathrm{bM}_{1}$, considerando a relação $M_{2}(t)=\frac{1}{\beta-(\lambda+\alpha)}\left(M_{1}(t)-\alpha C e^{-\beta t}\right)$, obtida dividindo-se (4) por (5) e considerando $\beta \approx 0$, indica a taxa de passagem do metal do solo para a raiz. Analogamente, o valor de $b$ do modelo linear $\mathrm{a}+\mathrm{bX}$, em que: $\mathrm{X}=\mathrm{M}_{2}$ e $\mathrm{Y}=\mathrm{M}_{3}$, que indicam a taxa de passagem do metal da raiz à parte aérea da cana, cujos valores de $b$ encontrados correspondem, respectivamente, aos parâmetros $\alpha$ e $\beta$ do modelo de compartimentos aqui proposto. Já no parâmetro $\lambda$, esse valor inicial foi conseguido pelo Experimento 1, com modelos lineares de regressão, para $\mathrm{Y}=$ teor de metal no solo e $\mathrm{X}=$ tempo de incubação do metal no solo, cujo valor do parâmetro $b$ desse modelo linear indica a taxa de decaimento do metal no solo. Os ajustes não-lineares para a estimativa dos parâmetros foram realizados para cada concentração de metal adicionada ao solo, que é o valor do parâmetro $C$ do modelo, isto é, quantidade do metal existente no CLU mais a quantidade de metal adicionada em forma de solução (CLU enriquecido). Além disso, esse ajuste foi realizado para cada grupo de solo (A e B) e para cada metal $(\mathrm{Cd}, \mathrm{Cu}, \mathrm{Ni}$ e $\mathrm{Pb}$ ). Como os dados obtidos no Experimento $1\left(\mathrm{M}_{1}, \mathrm{M}_{2}\right.$ e $\left.\mathrm{M}_{3}\right)$ foram coletados após 60 dias de plantio, o valor do $\mathrm{t}$ para ajuste dos modelos aos dados reais foi 0,1644 anos, e, como primeiro passo, foram determinados valores para $\alpha+\beta$, mediante a solução 1 :

$$
\begin{aligned}
& \mathrm{M}_{1}(\mathrm{t})=\mathrm{C} \mathrm{e}^{-(\lambda+\alpha) \mathrm{t}} \\
& \text { Considerando-se: } \alpha+\beta=\mathrm{x} \\
& \text { e resolvendo } x=\frac{\ln C-\ln M_{1}}{t}
\end{aligned}
$$

Após obtidos os valores de $\mathrm{x}(\mathrm{x}=\alpha+\beta)$ para cada parâmetro $\mathrm{C}$, em cada grupo de solo (A e B) e para cada metal pesado estudado, chegou-se, pela resolução de um sistema de equações, a um modelo de $M_{3}$ em função da variável $M_{2}$ (Solução 4), o qual foi ajustado para a estimativa do parâmetro $\alpha$ :

$$
\text { Solução 4: } \mathrm{M}_{3}=\frac{\alpha \mathrm{C}}{\lambda+\alpha}\left(1-\mathrm{e}^{-(\lambda+\alpha) t}\right)-\mathrm{M}_{2}
$$


Após estimados os valores de $\alpha$, resolveu-se a equação de $M_{2}$, isolando-se $\beta$ (solução 5) para a estimativa desse parâmetro em função de valores reais de $M_{2}$ :

$$
\text { Solução 5: } \beta=\frac{(\lambda+\alpha) \mathrm{M}_{2}+\alpha \mathrm{Ce}^{-(\lambda+\alpha) t}}{\mathrm{M}_{2}+\alpha \mathrm{Ct}}
$$

\section{RESULTADOS E DISCUSSÃO}

Com os valores obtidos dos parâmetros $\lambda, \alpha$ e $\beta$, foram realizadas simulações para cada nível de metal aplicado ao solo (C), em agrupamentos, e para cada metal, utilizando-se das soluções 1; 2 e 3 para a determinação de valores preditos de $\mathrm{M}_{1}, \mathrm{M}_{2}$ e $\mathrm{M}_{3}$ em várias épocas ( $\mathrm{t}$ ), para a estimativa do comportamento do metal pesado nas diferentes partes da cana-de-açúcar no tempo.

O comportamento de cada metal, simulado para $\mathrm{C}_{1}$, nos solos do grupo $\mathrm{A}$, para os teores de $\mathrm{M}_{1}, \mathrm{M}_{2}$ e $\mathrm{M}_{3}$, desde o momento da aplicação do CLU enriquecido, até dois anos após essa aplicação, apresenta queda rápida do teor de metal no compartimento solo, que é explicado pelos processos de adsorção, fixação e complexação dos metais (ADRIANO, 1986), comportamento do metal na raiz, começando do zero, com a presença de um ponto máximo (tempo onde é máximo o teor de metal na raiz), e crescimento do teor de metal no compartimento parte aérea da cana-deaçúcar, ficando esse teor, após determinado tempo, constante.

Nas Figuras 2A e 2B, observam-se baixas concentrações de $\mathrm{Cd}$ e de $\mathrm{Cu}$ no solo, que favorece a adsorção maior aos complexos organominerais (ADRIANO, 1986) que, nesse grupo, têm controle de solubilidade dos metais influenciado pelas reações associada à matéria orgânica e aos sesquióxidos (KABATA-PENDIAS \& PENDIAS, 1985).
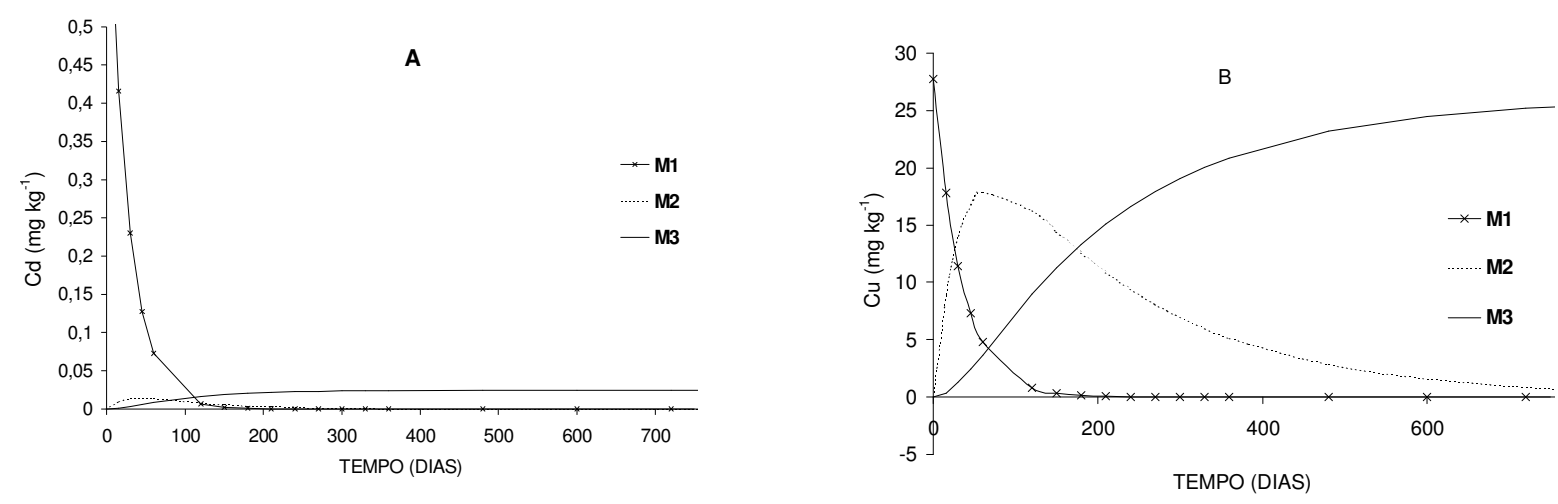

FIGURA 2. Modelo compartimental para o cádmio (A) e cobre (B) em solos A, sob adubação do nível 1 de composto de lixo urbano (CLU).

Na Figura 3A, notou-se que o teor total de $\mathrm{Pb}$ acumulou no solo na superfície, mas a fração solúvel é muito pequena, forma complexo organometálicos estáveis com a matéria orgânica e precipita como hidróxido, fosfato ou carbonatos (ADRIANO, 1986).

$\mathrm{Na}$ Figura 3B, nota-se que há decaimento de Ni no solo, que pode ser explicado pela imobilização pela matéria orgânica. 

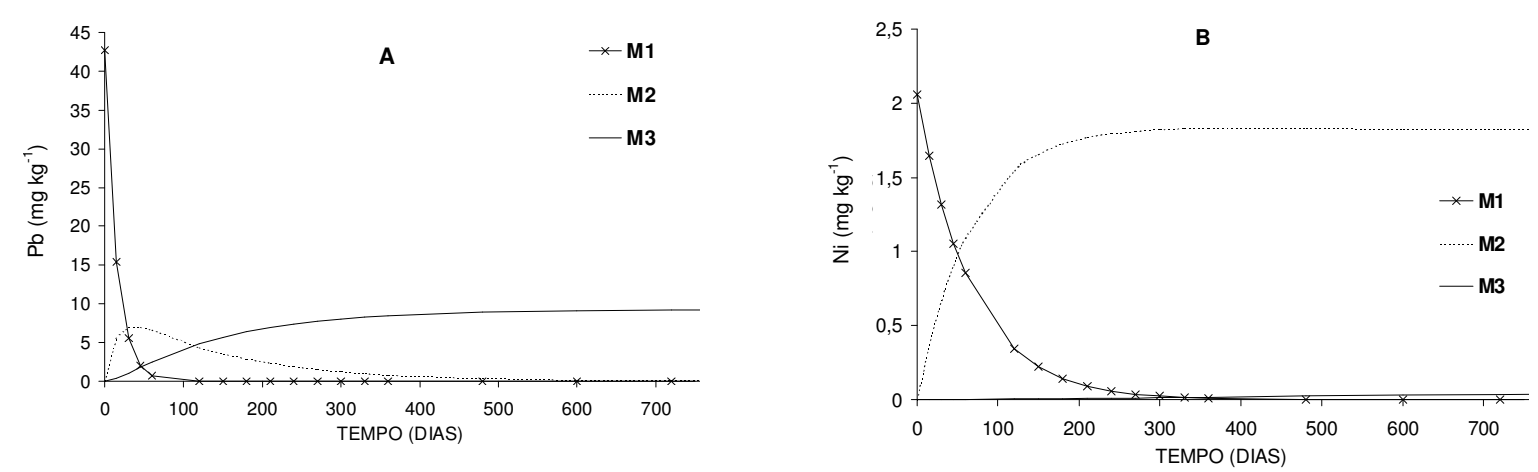

FIGURA 3. Modelo compartimental para o chumbo (A) e níquel (B) em solos A, sob adubação do nível 1 de composto de lixo urbano (CLU).

\section{Primeiro ponto crítico: $M_{1}=0$}

Quando $t$ tende ao infinito, $\mathrm{M}_{1}$ tende a zero, ou seja, a quantidade de metal pesado em todos os solos tende à extinção. Na curva para os teores de $\mathrm{Pb}$ nos solos da classe $\mathrm{A}$, apresenta-se rápida queda, chegando à extinção antes de 200 dias após adubado, e nos solos da classe B, essa queda é mais lenta, ficando entre 200 dias a um ano, após adubado com CLU. Para o Ni, observou-se queda acentuada apenas quando o solo é adubado com nível 1, chegando esse teor à extinção no compartimento um ano após a adubação de CLU, enquanto, nos outros níveis, esse tempo pode ultrapassar três anos (queda muito lenta), assim como o Cd nos níveis 4 e 5 dos solos da classe B, e o restante chegou a zero após 400 dias, aproximadamente. Para o $\mathrm{Cu}$, a queda de $\mathrm{M}_{1}$ foi mais lenta quando utilizado o nível 5 nos solos da classe B, chegando à zero, próximo de 600 dias após adubação de CLU, no nível 1, próximo aos 200 dias e nos demais, próximo aos 365 dias.

\section{Segundo ponto crítico: $M_{2}=$ máximo}

O ponto de máximo de $M_{2}$ acontece quando: $\alpha M_{1}=\beta M_{2}$. Então, o tempo ( $\left.t^{*}\right)$ onde ocorre o maior valor de metal na raiz da planta é dado por:

$$
t^{*}=\frac{\ln \frac{\beta}{\lambda+\beta}}{-\beta+\lambda+\alpha}
$$

As condições de contorno foram: $\mathrm{t}>\mathrm{t}^{*} \rightarrow \mathrm{M}_{2}$ é decrescente $; \mathrm{t}=\mathrm{t}^{*} \rightarrow \mathrm{M}_{2}$ é máximo; $\mathrm{t}<\mathrm{t}^{*} \rightarrow \mathrm{M}_{2}$ é crescente. Por esse método, podem-se analisar os metais pelo máximo teor que chegará à raiz da planta, a partir do qual começará a decrescer. Observou-se forte interferência das propriedades dos solos na absorção do metal pelo sistema radicular da planta, uma vez que tal processo ocorreu em ordem inversa aos valores de óxidos de ferro e teores de argila; isto é, quanto menores esses valores, maior a absorção de $\mathrm{Cd}$ e Ni pelas raízes das plantas.

Os solos foram divididos em classes A e B. Na classe A, enquadram-se os solos LV e B, e na classe B, os solos PL e PV. Foram feitos estudos preliminares de correlações lineares (valor $r$ ) entre as variáveis concentração de metal no solo e na raiz da cana-de-açúcar para conhecimento da dependência existente entre elas; isto é, se a variação na concentração de metal na raiz acompanharia proporcional ou inversamente proporcional a variação da concentração de metal no solo, sendo observado que: solos classe $\mathrm{A}$ - $\mathrm{Cd}(0,901), \mathrm{Cu}(0,750), \mathrm{Ni}(0,925)$ e $\mathrm{Pb}(0,908)$ e os solos classe B - Cd $(0,540), \mathrm{Cu}(0,955), \mathrm{Ni}(0,984)$ e $\mathrm{Pb}(0,989)$. Ficou evidente o relacionamento diretamente proporcional entre as variáveis, mas, de acordo com McBRIDE(1995), somente utilizando-se dos modelos mais complexos seria possível acompanhar as dinâmicas dessas variáveis no tempo e entre si, exceto pelo $\mathrm{Pb}$ que fica pouco disponível no solo. No teor de chumbo, não houve diferenças significativas entre os níveis, e o ponto máximo ocorre, em média, aos 49 dias após a adubação para os solos da classe A e aos 60 dias para os solos classe B. Uma 
fração muito pequena do $\mathrm{Pb}$ adicionado ao solo foi encontrada nas formas solúvel e trocável, consideradas disponíveis às plantas, na ordem de 1,6 a 8,0\% (ADRIANO, 1986).

Para $\mathrm{Ni}$, os solos $\mathrm{B}$ mostraram crescimento mais lento de metal na raiz, e o ponto máximo ocorreu, em média, aos 234 dias, com teor de $30 \%$ do Ni adicionado ao solo em forma de CLU enriquecido; e nos solos A, 128 dias, com teor máximo de 32\%. Em solos em que ocorre a correção de sua acidez com calagem, esse teor máximo reduz para ordem de 5\% (ADRIANO, 1986). Os menores valores estão nos níveis 1 , mas os teores de $M_{2}$ não são diretamente proporcionais aos níveis. Nos níveis 1, esses teores na raiz chegam a zero, próximo aos 500 dias, enquanto, nos outros níveis, ultrapassam os três anos, que são resultados similares aos obtidos por TIPPING (1998). Para Cd, os solos B mostraram crescimento mais lento, e a média do tempo para $M_{2}$ atingir o ponto máximo é 156 dias, e para os solos A é de 120 dias, cujo ponto máximo é de $26 \%$ para ambos os solos. Para $\mathrm{Cu}$, o tempo variou de 52 a 90 dias. No nível 1, ocorreu maior velocidade de transferência de $\mathrm{Cd}$ para a raiz, chegando ao ponto máximo em 48 dias nos solos A e 85 dias nos solos B e, em todos os casos, $M_{2}$ só chega a zero após, aproximadamente, dois anos. A passagem máxima de $M_{2}$ para o metal Cu fica, em média, $45 \%$ de $\mathrm{C}$, com exceção dos níveis 4 e 5 (A) que ficam em torno de $27 \%$ de $C$.

\section{Terceiro ponto crítico: ponto em que $M_{3}$ se estabiliza}

$\mathrm{O}$ valor de $\mathrm{M}_{3}$ é crescente e se estabiliza em $(\alpha \mathrm{C}) /(\lambda+\alpha)$. Em estudos preliminares de correlação linear (valor $r$ ) entre as variáveis concentração de metal na raiz e na parte aérea da canade-açúcar, para conhecimento da dependência existente entre elas; isto é, a disponibilidade do metal é relacionada a sua concentração em solução do solo, mas é condicionada pelas características da sua fração sólida (VIJVER et al., 2003), que reflete nos processos de precipitação, de adsorção e de complexação. Tais características da fração sólida explica as distintas correlações entre o metal no solo por agrupamento com as plantas, como observado nos valores de r: em solos da classe A - Cd $(0,893), \mathrm{Cu}(0,382), \mathrm{Ni}(0,846)$ e $\mathrm{Pb}(-0,520)$ e da classe $\mathrm{B}$ $\mathrm{Cd}(0,922), \mathrm{Cu}(0,594), \mathrm{Ni}(0,931)$ e $\mathrm{Pb}(-0,232)$. Os teores de $\mathrm{Cd}, \mathrm{Cu}$ e $\mathrm{Ni}$ nas partes da planta foram diretamente proporcionais e o $\mathrm{Pb}$ inversamente proporcional, que concorda com os resultado de DUDKA et al., (1996).

$\mathrm{O}$ teor de $\mathrm{Pb}$ na parte aérea $\left(M_{3}\right)$ está praticamente estabilizado e demostrando uma barreira natural da planta a sua translocação, o que corresponde, na classe de solo A, que o teor de $M_{3}$ vai permanecer em torno da média máxima de $19 \%$ do teor de $\mathrm{Pb}$ adicionado ao solo $(C)$, e para os solos da classe $\mathrm{B}$, de $35 \%$ de $C$. Nota-se, ainda, que os solos B proporcionaram maior translocação do metal para a parte aérea, e suas curvas de $M_{1}$ e $M_{2}$ apresentaram quedas mais lentas. Para $\mathrm{Ni}$, não foram observadas diferenças desse metal quando comparados os solos $\mathrm{A}$ e $\mathrm{B}$, mas deve ser tomado muito cuidado, pois o teor de Ni na parte aérea, quando se estabiliza, fica em torno de $99 \%$ do teor adicionado ao solo (CLU enriquecido) em todos os níveis, acumulando-se nas raízes. A translocação de Ni das raízes para a parte aérea foi pequena no primeiro ano, além disso, estabiliza-se depois de aproximadamente três anos de adubado.

A quantidade de $\mathrm{Cd}$ na parte aérea da cana, nos níveis 1 dos solos $\mathrm{A}$ e $\mathrm{B}$, foi bem baixo, em torno de $4 \%$ do teor de $\mathrm{Cd}$ adicionado $(C)$, nos níveis 2 do solo $\mathrm{A}$, e 4 e 5 dos solos $\mathrm{B}$, explicado pela diferença nos conteúdos de matéria orgânica e de sesquióxidos (ADRIANO, 1986); esse teor na parte aérea chega a $99 \%$ de $C$, enquanto nos demais tratamentos fica em torno de $50 \%$ do teor adicionado. $\mathrm{O}$ tempo onde $\mathrm{M}_{3}$ se estabiliza nesse valor, fica em torno de dois anos, e para os níveis 4 e 5 dos solos B, ultrapassam os três anos (crescimento lento).

$\mathrm{O}$ teor de $\mathrm{Cu}$ na parte aérea é preocupante, pois ele se estabiliza, com exceção dos níveis 4 e 5 dos solos da classe A, próximo a $99 \%$ do teor adicionado $(C)$, ficando esses outros dois em torno de $60 \%$ de $C$. O cuidado deve ser ainda maior, pois esse compartimento atinge esses altos teores, onde se estabilizam, próximo aos 365 dias após adubação, que é a época de corte da cana.

Conforme análises dos parâmetros e pontos críticos em relação ao limites da legislação (Tabela 1), pode-se chegar a algumas conclusões que servirão como base na formulação de normas 
de utilização e recomendação do CLU na cultura da cana-de-açúcar. O metal mais móvel no sistema solo-planta, mediante análise dos modelos ajustados, é o $\mathrm{Ni}$, e o menos móvel foi o $\mathrm{Pb}$, chegando na parte aérea com média de $99 \%$ e $27 \%$ dos metais adicionados, respectivamente (Tabela 2). No caso de Pb, ADRIANO (1986) destaca que existem espécies capazes de bloquear o movimento do metal no acoplasto, diminuindo o transporte para o xilema da raiz para parte aérea, que pode ser complexado na forma de compostos insolúveis na parede celular e membrana (BENAVIDES et al., 2005), o que explica a correlação negativa de $\mathrm{Pb}$ entre as raízes e a parte aérea.

Ressalta-se que a cultura de cana-de-açúcar possui uma barreira diferenciada para bloquear a transferência dos metais no sistema planta, cujas raízes acumulam e imobilizam-no no xilema, retratado no modelo proposto, que não é a mesma realidade para as hortaliças de consumo direto sem qualquer processamento (ADRIANO, 1986; SILVA et al. 2002). De acordo com KABATAPENDIAS \& PENDIAS (1985), cada metal acumulado na planta transloca-se diferentemente da raiz para a parte aérea, que seriam: o $\mathrm{Ni}$ e o Zn distribuídos uniformemente entre raízes e parte aérea; o $\mathrm{Cu}$ e o $\mathrm{Cd}$, usualmente com maior teor nas raízes e com quantidades moderadas a grande na parte aérea, e o $\mathrm{Pb}$ nas raízes, como muito pouco na parte aérea, sendo essa redistribuição muito variável em função da espécie.

$\mathrm{Na}$ Tabela 2, nota-se que o metal que apresenta maior ponto máximo na raiz em relação ao teor adicionado, é também o $\mathrm{Cu}$, com média de $41 \%$, no tempo médio de 75 dias. Porém, o que cresce mais rápido na raiz é o $\mathrm{Pb}$, o qual, em média, aos 55 dias, chega ao seu teor máximo, que fica em torno de $15 \%$ do nível adicionado ao solo. O metal mais lento é ainda o $\mathrm{Ni}$, que chega ao ponto máximo, em média, aos 180 dias, com o teor máximo de $31 \%$ de $C$.

TABELA 2. Resultados médios nos pontos críticos por compartimento estimados pelos modelos ajustados de transferências de metais pesados no sistema solo-cana adubados com CLU.

\begin{tabular}{cccccc}
\hline \multirow{2}{*}{ Metal } & $\begin{array}{c}\text { "t" em que } \\
\mathrm{M}_{1 \rightarrow 0}\end{array}$ & $\begin{array}{c}\text { "t” em que } \mathrm{M}_{2} \text { é } \\
\text { máximo }\end{array}$ & $\begin{array}{c}\mathrm{M}_{2} \text { máximo } \\
(\% \text { de } \mathrm{C})\end{array}$ & $\begin{array}{c}\text { "t” em que } \mathrm{M}_{3} \\
\text { se estabiliza }\end{array}$ & $\begin{array}{c}\mathrm{M}_{3} \text { máximo } \\
(\% \text { de } \mathrm{C})\end{array}$ \\
\hline $\mathrm{Cd}$ & 600 dias & 138 dias & $26 \%$ & $>600$ dias & $55 \%$ \\
$\mathrm{Cu}$ & 400 dias & 181 dias & $41 \%$ & $<365$ dias & $92 \%$ \\
$\mathrm{Ni}$ & 3 anos & 55 dias & $31,3 \%$ & $>600$ dias & $99 \%$ \\
$\mathrm{~Pb}$ & 200 dias & 75 dias & $14,5 \%$ & $<365$ dias & $27 \%$ \\
\hline
\end{tabular}

\section{CONCLUSÕES}

Os modelos matemáticos de transferência de metais pesados são específicos para cana-deaçúcar, refletindo mecanismos de bloqueio próprios de passagem dos metais no sistema soloraízes-parte aérea. No solo, o metal mais preocupante foi o $\mathrm{Ni}$, seguido do $\mathrm{Cd}$ e $\mathrm{Cu}$, pois demoraria três anos para retornar aos valores originais de disponibilidade no solo, possuindo efeito residual na soqueira. $\mathrm{O} \mathrm{Pb}$ foi de decaimento mais rápido no solo pela forte interação com óxidos de ferro e alumínio e argila. Na cana-de-açúcar de maior preocupação é com o Cu, que além de muito móvel, possui alta velocidade de passagem se não estiver imobilizado na matéria orgânica do solo.

\section{AGRADECIMENTOS}

Para aqueles que geraram os dados: Carlos Alberto Silva, André Luiz Ramalho e Cláudia Maria Villar Caldeira, e à Fapesp, pelo apoio financeiro.

\section{REFERÊNCIAS}

ADRIANO, D.C. Trace elements in the terrestrial environmental. New York: Springer Verlag, 1986. 533 p. 
ALVES, W.L. Compostagem e vermicompostagem no tratamento de lixo urbano. Jaboticabal: FUNEP, 1996. 46 p.

BAILEY, S.E.; OLIN, T.J.; BRICA, R.M.; ADRIN, D.D. A review of potential low cost sorbents for heavy metals. Walter Research, New York, v.33, n.11, p.2469-79, 1999.

BASSANEZI, R.C.; FERREIRA JÚNIOR, W. C. Equações diferenciais com aplicações. São Paulo: Editora Harbra, 1988. 572 p.

BENAVIDES, M.P.; GALLEGO, S.M.; TOMARO, M.L.C Cadmium toxicity in plants. Brazilian of Journal Plant Physiolology, Londrina, v.17, n.1, p. 21-34, 2005.

CETESB. COMPANHIA DE TECNOLOGIA DE SANEAMENTO AMBIENTAL. Relatório de estabelecimento de valores orientadores para proteção da qualidade de solos e água no Estado de São Paulo. São Paulo, 2001. 247 p. Disponível em: www.cetesb.sp.gov.br. Acesso em: 10 out. 2005.

DUDKA, S.; PIOTROWSKA, M; TERELAK, H. Transfer of cadmiun, lead and zinc from industrially contaminated soil to crop plants: a field study. Environmental Pollution, Kidlington, v.94, n.2, p.181-8, 1996.

EPA. ENVIRONMENTAL PROTECTION AGENCY. A guide to teh biosolid risk assessments for the part 503 rule. Washington, 1995. (EPA832-B-93-005).

KABATA-PENDIAS, A.; PENDIAS, H. Trace elements in soils and plants. Boca Raton: CRC Press, 1985. 315 p.

McBRIDE, M.B. Toxic metal accumulation from agricultural use of sludge: are USEPA regulations protective ? Journal Environmental Quality, Madison, v.24, n.1, p.5-18, 1995.

MEERS, E.; UNAMUNO, V.R.; LAING, G. DU; VANGRONSVELD, J.VANBROEKHOVEN, K.; SAMSON, R.; DIELS, L.; GEEBELEN, W.; RUTTENS, A.; VANDEGEHVCHTE, M.; TACK, F.M.G. $\mathrm{Zn}$ in the soil solution of unpolluted and polluted soils as affected by soil característics. Geoderma, Weergeven, v.130, n.1, p.15-27, maio 2006.

SAS INSTITUTE. SAS user's guide: statistics version 6. 4.ed. Cary, 1990. 168 p.

SILVA, F.C. da. (Org.). Manual de análise químicas de solos, plantas e fertilizantes. Brasília: EMBRAPA, 1999. v.1. 370 p.

SILVA, F.C.; CALDEIRA, C.V.; GOMES, P.C.; BERGAMASCO, A.F. Uso agrícola de composto de lixo: estudo da transferência de metal pesado no sistema solo-cana. Campinas: Embrapa Informática Agropecuária, 2000b. 5 p. (Comunicado Técnico, 12).

SILVA, F.C.; SILVA, C.A.; BERGAMASCO, A.F.; RAMALHO, A.L. Uso agrícola de composto de lixo: efeito do tempo de incubação solo/resíduo na disponibilidade de metal pesado. Campinas: Embrapa Informática Agropecuária, 2000a. 11 p. (Comunicado Técnico, 10).

SILVA, F.C. da; BERTON, R.S.; CHITOLINA, J.C.; BALLESTERO, S.D. Recomendações técnicas para o uso agrícola do composto de lixo urbano no Estado de São Paulo. Campinas: Embrapa Informática Agropecuária, 2002. 17 p. (Circular Técnica, 3).

TIPPIJNG, E. Humic ino-biding model IV: na improved descrition on the interaction of protons and metal ions with humic substances. Aqueous Geochemistry, Hynes, v.4, n.1, p.3-48, 1998.

VIJVER, M.; JAGER, T.; POSTHUMA, L.; PEIJNENBURG, W. Metal uptake from soilsediment mixtures by larval of teobrio monitor (L.) (coleoptera). Ecotoxicology and Environmental Safety, Weergeven, v.54, p.277-89, 2003. 